\title{
The Moments in Control: a tool for Analysis, Reduction and Design
}

\author{
Abdelmadjid Bentayeb, Nezha Maamri, Jean-Claude Trigeassou
}

\begin{abstract}
In this paper we present a new method of model reduction via the moments. The reduction technique is composed of two steps, the first one consists on using the Least Squares linear optimization algorithm to minimize a cost function representing the norm 2 of the error between different moments of the full order function and the reduced model. This solution represents an initialization of the second step algorithm which is based a Non Linear Programming minimizing a new criterion composed of the cost function of the first step and an equality constraint.
\end{abstract}

Keywords: Moments, model reduction, optimization.

\section{Introduction}

During the last 30 years many design techniques like $\mathcal{H}_{\infty}$ [10] was elaborated in order to obtain better performances of controlled plants but once the performances objectives ensured, implementation problems appears because most of industrial applications still use a simple controllers structure like PID (Proportional Integral Derivative) [1][10]; so the aim after the synthesis step is to find a reduced order controller easy to use and to implement, this reduced controller must ensure as possible the same performances of the full order controller[9].

Since reduction can not ensure the same performances of the full order controller in all frequencies, it is reasonable to specify a frequency range to proceed to the reduction [3][4].

There is many model reduction techniques like Balanced Truncation [11] and Optimal Hankel norm approximation [10]; every method differs from the other one by the importance accorded to d.c gain or to middle and high frequencies.

Our reduction model method is original, it is based on the notion of moments which is a description of linear time invariant system around a given pulsation [12]. The methodology is composed of two steps, in the first one a cost function representing the norm 2 of the error between different frequency moments of the full order system and the reduced order one is minimized. Some parameters are imposed a priori to obtain a linear criterion, so the parameters of the reduced model are computed using Least Squares algorithm [2][5].

The solution obtained from the step one is used to initialize the Non Linear Programming algorithm of the second step in order to minimize a criterion composed of the cost function of the first step and an equality constraint between the first time moments of the full order system and the reduced model to ensure low frequencies performances [7].

The paper is organized as follows: in section 2, we present definitions and computing methods of time and frequency moments; in section 3, we develop the model reduction technique by presenting the principle, the Linear optimization and the Non Linear optimization and section 4 is devoted to conclusions. Notice that the illustrative examples are presented in section 3 .

\section{The Moments}

Let us consider a linear SISO system, characterized by its transfer function $G(s)$ analytic in the RHP plan (.i.e $\operatorname{Re}(s)>0$ ) and let $g(t)$ be its impulse response:

$$
G(s)=\int_{0}^{\infty} g(t) e^{-s t} d t
$$


The transfer function is given by the following state space (not necessary minimal) realization:

$$
G(s) \stackrel{s}{=}\left[\begin{array}{l|l}
A & B \\
\hline C & D
\end{array}\right]=C(s I-A)^{-1} B+D
$$

where $A \in \mathscr{R}^{n \times n}, B \in \mathscr{R}^{n \times 1}, C \in \mathscr{R}^{1 \times n}$ and $D \in \mathscr{R}^{1 \times 1}$.

\subsection{Time moments}

By expanding $e^{-s t}$ in Taylor series in the vicinity of $s=0$, we get:

$$
\begin{gathered}
G(s)=\int_{0}^{\infty} \sum_{n=0}^{\infty}(-1)^{n} s^{n} \frac{t^{n}}{n !} g(t) d t \\
G(s)=\sum_{n=0}^{\infty}(-1)^{n} \mathcal{A}_{n}(g) s^{n}
\end{gathered}
$$

where:

$$
\mathcal{A}_{n}(g)=\int_{0}^{\infty} \frac{t^{n}}{n !} g(t) d t
$$

$\mathcal{A}_{n}$ : represents the $n^{\text {th }}$ order moment of $g(t)$.

Remark 1 . The time moments $\mathcal{A}_{n}$ give a description of the system at low frequencies.

- $\mathcal{A}_{0}(g)$ represents the area or the d.c gain of $g(t)$.

- $\mathcal{A}_{1}(g)$ defines mean time of $g(t)$.

- $\mathcal{A}_{2}(g)$ deals with the 'dispersion' of $g(t)$ around its mean time,...etc [2][5][7]

\subsection{Frequency moments}

Let consider the variable $s=j \omega$. By expanding $e^{-s t}$ in Taylor series in the vicinity of $s_{0}=j \omega_{0}$, we get:

$$
G(j \omega)=\sum_{n=0}^{\infty}(-1)^{n}\left(j \omega-j \omega_{0}\right)^{n} \underline{\mathcal{A}}_{n, \omega_{0}}(g)
$$

with:

$$
\underline{\mathcal{A}}_{n, \omega_{0}}(g)=\int_{0}^{\infty} \frac{t^{n}}{n !} e^{-\left(s-s_{0}\right) t} g(t) d t
$$

Remark 2. Like the time moments, the frequency moments describe the system around $\omega=\omega_{0}$ :

- $\underline{\mathcal{A}}_{0, \omega_{0}}$ represents $G(j \omega)$ at $\omega=\omega_{0}$.

- $\underline{\mathcal{A}}_{0, \omega_{0}}-j\left(\omega-\omega_{0}\right) \underline{\mathcal{A}}_{1, \omega_{0}}$ permits to enlarge the previous approximation around $\omega=\omega_{0}$.

Notice that the moments $\underline{\mathcal{A}}_{n, \omega_{0}}$ are complex and if $\omega_{0}=0$, we recover the time moments of the system (i.e $\left.\underline{\mathcal{A}}_{n, 0}=\mathcal{A}_{n}\right)[7]$. 


\subsection{Computing the moments using state space realization}

\section{Time moments}

Using the following equality:

$$
(s I-A)\left(-A^{-1}-s A^{-2}-s^{2} A^{-3}-\cdots-\right)=I \Rightarrow(s I-A)^{-1}=-\sum_{n=0}^{\infty}\left(s^{n} A^{-(n+1)}\right)
$$

an from (2) and (4), we can write:

$$
G(s)=-C\left(\sum_{n=1}^{\infty} s^{n} A^{-(n+1)}\right) B+\left(-C A^{-1} B+D\right)
$$

so:

$$
\mathcal{A}_{0}(g)=-C A^{-1} B+D \quad \text { and } \quad \mathcal{A}_{n}(g)=(-1)^{n+1} C A^{-(n+1)} B, \quad(n=1 \cdots \infty)
$$

\section{Frequency moments}

Realizing a variable change $\mu=j \omega-j \omega_{0}$, equation (6) becomes:

$$
G(\mu)=\sum_{n=0}^{\infty}(-1)^{n}(\mu)^{n} \underline{\mathcal{A}}_{n, \omega_{0}}(g)
$$

and (2):

$$
G(\mu)=C\left(\mu I-\left(-j \omega_{0} I+A\right)\right)^{-1} B+D
$$

so, we get:

$$
\begin{gathered}
\underline{\mathcal{A}}_{0, \omega_{0}}(g)=-C\left(-j \omega_{0} I+A\right)^{-1} B+D \\
\underline{\mathcal{A}}_{n, \omega_{0}}(g)=(-1)^{n+1} C\left(-j \omega_{0} I+A\right)^{-(n+1)} B, \quad(n=1 \cdots \infty)
\end{gathered}
$$

\section{Model Reduction}

The purpose of model reduction is, starting from a real system, to find a reduced model making it possible as well as possible to approximate it in a given frequency range.

\subsection{Principle}

Let $G(s)$ be a nominal transfer function of high order:

$$
G(s)=\frac{b_{0}+b_{1} s+\cdots+b_{m} s^{m}}{a_{0}+a_{1} s+\cdots+a_{n-1} s^{n-1}+s^{n}}, \text { with } m \leq n
$$

and the parameters vectors is:

$$
\underline{\theta}^{T}=\left[a_{0} a_{1} \cdots a_{n-1} b_{0} b_{1} \cdots b_{m}\right]
$$

We define a reduced structure:

$$
G_{r}(s)=\frac{b_{0 r}+b_{1 r} s+\cdots+b_{m r} s^{m r}}{a_{0 r}+a_{1 r} s+\cdots+a_{(n-1) r} s^{n r-1}+s^{n r}}, \text { with } m r \leq n r
$$




\subsection{Linear Optimization}

Let consider the following reduced structure [6]:

$$
G_{r}(s)=\frac{N_{r}(s)}{D_{r}(s)}
$$

in linear optimization, we consider that the reduced denominator $D_{r}(s)$ is fixed a priori and only the numerator parameters have to be optimized. As it is evoked before the reduced model try to ensure:

$$
G_{r}(s)=G(s)
$$

in a frequency range evidently.

Equation (19) can be written:

$$
\frac{b_{0 r}+b_{1 r} s+\cdots+b_{m r} s^{m r}}{D_{r}(s)}=G(s)=\frac{1}{D_{r}(s)}\left(\sum_{k=0}^{m r} b_{k} s^{k}\right)
$$

using the moments, the previous equation becomes:

$$
\left(\sum_{k=0}^{m r} b_{k} s^{k}\right)\left(\sum_{k=0}^{\infty}(-1)^{k} \underline{\mathcal{A}}_{k, \omega_{0}} s^{k}\right)=\left(\sum_{k=0}^{\infty}(-1)^{k} \underline{\mathcal{A}}_{k, \omega_{0}}^{\prime} s^{k}\right)
$$

where:

$$
\underline{\mathcal{A}}_{k, \omega_{0}} \text { represent the } n^{\text {th }} \text { order frequency moment of } \frac{1}{D_{r}(s)}
$$

and

$$
\underline{\mathcal{A}}_{k, \omega_{0}}^{\prime} \text { represent the } n^{t h} \text { order frequency moment of } G(s)
$$

After truncation until $n r$ and by equalizing the terms of the same power in $s$, the equation (21) can be written as follow:

$$
\left[\begin{array}{ccccc}
\underline{\mathcal{A}}_{0, \omega_{0}} & 0 & \cdots & \cdots & 0 \\
-\underline{\mathcal{A}}_{1, \omega_{0}} & \underline{\mathcal{A}}_{1, \omega_{0}} & 0 & \cdots & 0 \\
\vdots & \vdots & \ddots & \cdots & \vdots \\
\underline{\mathcal{A}}_{n r, \omega_{0}} & \underline{\mathcal{A}}_{n r-1, \omega_{0}} & \cdots & \cdots & \underline{\mathcal{A}}_{0, \omega_{0}}
\end{array}\right]\left[\begin{array}{c}
b_{0 r} \\
b_{1 r} \\
\vdots \\
b_{m r}
\end{array}\right]=\left[\begin{array}{c}
\underline{\mathcal{A}}_{0, \omega_{0}}^{\prime} \\
-\underline{\mathcal{A}}_{1, \omega_{0}} \\
\vdots \\
\underline{\mathcal{A}}_{n r, \omega_{0}}^{\prime}
\end{array}\right]
$$

which can be written as:

$$
\Phi_{n r, m r} \underline{\theta}_{r}=\Gamma_{n r}
$$

Let:

$$
\underline{\varepsilon}_{n r}=\Gamma_{n r}-\Phi_{n r, m r} \underline{\theta}_{r}
$$

be the error between the $m r^{\text {th }}$ first moments between the real system and the reduced one. Notice that the moments can be either temporal or frequency ones it depends on the frequency range chosen.

We want to determine $\underline{\theta}_{r}$ which minimize the following quadratic cost:

$$
J=\underline{\varepsilon}^{T} \underline{\varepsilon}
$$

Since the system given by (25) is linear, we can determine $\underline{\theta}_{r}$ using Least Squares method, so:

$$
\underline{\theta}_{r}=\left[\Phi_{n r, m r}^{T} \Phi_{n r, m r}\right]^{-1}\left[\Phi_{n r, m r}^{T} \Gamma_{n r}\right]
$$


Example 3. Let us consider the following transfer function:

$$
G(s)=\frac{1.042 s^{7}+21.77 s^{6}+206.5 s^{5}+1049 s^{4}+2583 s^{3}+1789 s^{2}+437.5 s+35}{s^{7}+22.38 s^{6}+228.3 s^{5}+1323 s^{4}+3832 s^{3}+6339 s^{2}+1995 s+157.5}
$$

we want to find a two states reduced model which approximates as well as possible $G(s)$ in low, medium and high frequencies. First let choose a reduced denominator, for our case the choice is:

$$
D_{r}(s)=(1+0.5 s)^{2}
$$

so the number of numerator's parameters to be computed will not exceed 3 and the number of moments used equals the number of parameters; we choose three pulsations for reduction:

$$
\omega_{0}=0 r d / s, \omega_{0}=0.5 r d / s \text { and } \omega_{0}=2 r d / s
$$

The parameters vector $\underline{\theta}_{r}^{T}=\left[b_{0 r} b_{1 r} b_{2 r}\right]$ for the three cases is:

$$
\begin{aligned}
& \underline{\theta}_{r}^{T}=\left[\begin{array}{lll}
0.2222 & 0.1852 & 2.9012
\end{array}\right], \text { for } \omega_{0}=0 \mathrm{rd} / \mathrm{s} \\
& \underline{\theta}_{r}^{T}=\left[\begin{array}{lll}
0.1805 & 0.5030 & 0.2990
\end{array}\right], \text { for } \omega_{0}=0.5 \mathrm{rd} / \mathrm{s} \\
& \underline{\theta}_{r}^{T}=\left[\begin{array}{lll}
0.2969 & 0.5255 & 0.3808
\end{array}\right], \text { for } \omega_{0}=2 \mathrm{rd} / \mathrm{s}
\end{aligned}
$$

the frequency response of the real system and reduced model for the three cases is given in (Figure 1).
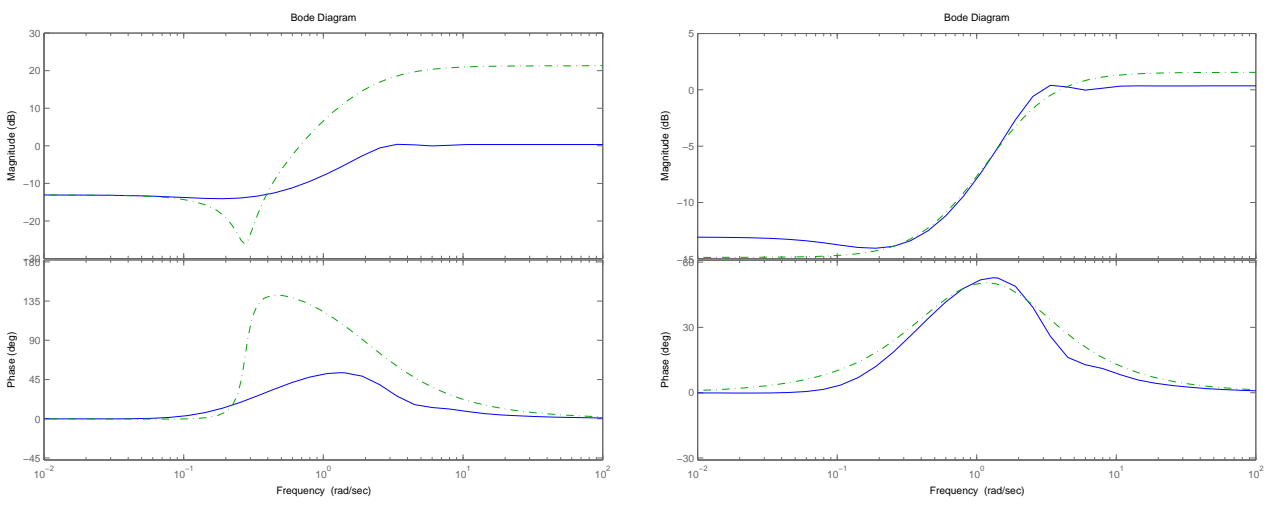

$$
G(s)(-), G_{r}(s)(-.-), \omega_{0}=0 r d / s \quad G(s)(-), G_{r}(s)(-.-), \omega_{0}=0.5 r d / s
$$

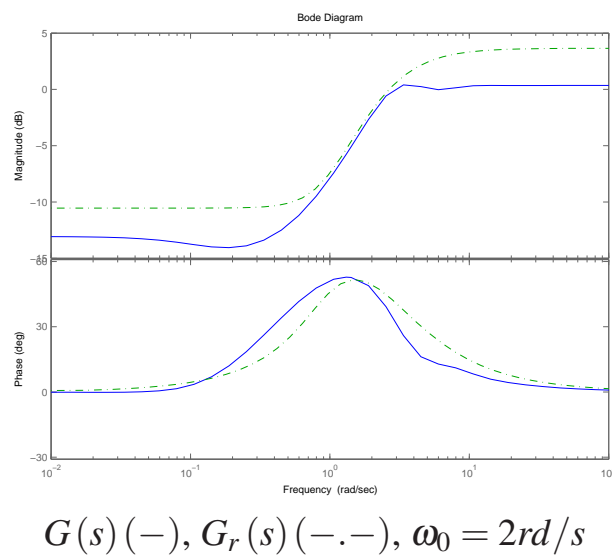

Figure 1: Frequency response of real system and reduced models 


\subsection{Non Linear Optimization}

It is clear that the fact of imposing the denominator of the reduced model, limits the optimization's performances, also if the reduction is needed in high frequencies, it is necessary to ensure at the same time the low frequencies behaviour.

In this part, we will optimize both poles and zeros of the reduced model or the parameters of numerator and denominator; a non linear optimization algorithm is used to minimize a quadratic cost between the moments of the real system and the reduced model including an equality constraint between the two first time moments in order to keep the same low frequencies performances.

\section{Principle}

Let us consider the parameters vector of a reduced model:

$$
\underline{\theta}_{r}^{T}=\left[a_{0 r} a_{1 r} \cdots a_{(n-1) r} b_{0} b_{1 r} \cdots b_{m r}\right]
$$

We want to find $\underline{\theta}_{r}^{T}$ which minimizes (27); for that we use Marquardt's algorithm [8] which is a good combination between rapidity and convergence.

\section{Algorithm principle}

Parameter estimation is performed using an iterative optimization procedure:

$$
\begin{gathered}
\hat{\underline{\theta}}_{i+1}=\underline{\hat{\theta}}_{i}-\left\{\left[J^{\prime \prime}+\lambda_{i} I\right]^{-1} . J^{\prime}\right\}_{\hat{\underline{\theta}}=\underline{\hat{\theta}}_{i}} \\
\left(\frac{\partial J}{\partial \underline{\theta}_{r}}\right)=J^{\prime}: \quad \text { the Gradient vector } \\
\left(\frac{\partial^{2} J}{\partial \underline{\theta}_{r}^{2}}\right)=J^{\prime \prime}: \quad \text { the Hessian matrix } \\
\lambda_{i}: \quad \text { coefficient to be adjusted }
\end{gathered}
$$

The initialization is given by the vector parameters emerged from the Least Squares optimization:

$$
\hat{\theta}_{0}=\hat{\theta}_{L S}
$$

\section{Computing $J^{\prime}$ and $J^{\prime \prime}$}

The calculation of the gradient and the hessian is crucial for the optimization procedure, we use parametric sensitivity function to calculate them:

$$
\begin{gathered}
J^{\prime} \approx-2 \sum_{n=0}^{N} \varepsilon_{n} \Theta_{n} \quad \text { and } \quad J^{\prime \prime} \approx 2 \sum_{n=0}^{N} \Theta_{n} \Theta_{n}^{T} \\
\Theta=\frac{d \underline{\mathcal{A}}_{n, \omega_{0}}\left(G_{r}\right)}{d \underline{\theta}_{r}}
\end{gathered}
$$

where:

$$
\frac{d \underline{\mathcal{A}}_{n, \omega_{0}}}{d \theta_{i}}=(-1)^{n+1}\left(\frac{d C}{d \theta_{i}} A_{\mu}^{-(n+1)} B-C A_{\mu}^{-(n+1)} \frac{d A_{\mu}^{(n+1)}}{d \theta_{i}} A_{\mu}^{-(n+1)} B+C A_{\mu}^{-(n+1)} \frac{d B}{d \theta_{i}}\right)
$$


where:

$$
\frac{d A_{\mu}^{(n+1)}}{d \theta_{i}}=\left(\frac{d A_{\mu}}{d \theta_{i}} A_{\mu}^{n}+A_{\mu} \frac{d A_{\mu}^{n}}{d \theta_{i}}\right) \quad \text { and } \quad A_{\mu}=\left(A-j \omega_{0} I\right)
$$

If we use a control or an observer canonical realization, (43) will be much easier to calculate. For Example, for control realization:

$$
\frac{d \underline{\mathcal{A}}_{n, \omega_{0}}}{d a_{i}}=(-1)^{n+1}\left(-C A_{\mu}^{-(n+1)} \frac{d A_{\mu}^{(n+1)}}{d a_{i}} A_{\mu}^{-(n+1)} B\right) \text { and } \frac{d \mathcal{A}_{n, \omega_{0}}}{d b_{i}}=(-1)^{n+1}\left(\frac{d C}{d b_{i}} A_{\mu}^{-(n+1)} B\right)
$$

where:

$$
\underline{a}_{r}=\left[\begin{array}{llll}
a_{0 r} & a_{1 r} \cdots a_{n r}
\end{array}\right] \quad \text { and } \quad \underline{b}_{r}=\left[\begin{array}{lll}
b_{0 r} & b_{1 r} \cdots b_{m r}
\end{array}\right]
$$

Now, we want to find $\underline{\theta}_{r}$ which minimizes (27) and ensures at the same time the following equality:

$$
F\left(\underline{\theta}_{r}\right)=\left\|\mathcal{A}_{0}(G)-\mathcal{A}_{0}\left(G_{r}\right)\right\|+\left\|\mathcal{A}_{1}(G)-\mathcal{A}_{1}\left(G_{r}\right)\right\|=0
$$

The optimization problem can be reformulated as:

$$
\min _{\underline{\theta}_{r}} J_{\text {const }} \text { with } J_{\text {const }}=J+\gamma F\left(\underline{\theta}_{r}\right)
$$

which is equivalent to: $\gamma$ represents the vector of Lagrange multipliers to be estimated.

To solve this problem, we can use the algorithm described in (36), by substituting $J$ by $J_{\text {const }}$, with:

$$
J_{\text {const }}^{\prime}=\left[\begin{array}{c}
\frac{\partial J_{\text {const }}}{\partial \theta} \\
\frac{\partial J_{\text {const }}}{\partial \gamma}
\end{array}\right], \quad J_{\text {const }}^{\prime \prime}=\left[\begin{array}{cc}
\frac{\partial^{2} J_{\text {const }}}{\partial \theta^{2}} & \frac{\partial^{2} J_{\text {const }}}{\partial \theta \partial \gamma} \\
\frac{\partial^{2} J_{\text {const }}}{\partial \theta \partial \gamma} & \frac{\partial^{2} J_{\text {const }}}{\partial \gamma^{2}}
\end{array}\right]
$$

Example 4. Let us take the same system given in Example 2:

$$
G(s)=\frac{1.042 s^{7}+21.77 s^{6}+206.5 s^{5}+1049 s^{4}+2583 s^{3}+1789 s^{2}+437.5 s+35}{s^{7}+22.38 s^{6}+228.3 s^{5}+1323 s^{4}+3832 s^{3}+6339 s^{2}+1995 s+157.5}
$$

the aim is to find a reduced order model of three states which approximate the real system around $\omega_{0}=$ $11 \mathrm{rd} / \mathrm{s}$ and have the same low frequencies behaviour; for that we will compare the results obtained from Least Squares, Non Linear Programming (NLP) and Non Linear Programming with equality constraint. The reduced model have the following structure:

$$
G_{r}(s)=\frac{b_{0 r}+b_{1 r} s+b_{2 r} s^{2}}{a_{0 r}+a_{1 r} s+s^{2}}
$$

of course for Least Squares optimization, the denominator is fixed a priori:

$$
D_{r}(s)=(1+0.5 s)^{2}
$$

The three reduced model are:

$$
\begin{array}{lr}
G_{r}(s)=\frac{1.019-1.698 s+0.6728 s^{2}}{(1+0.5 s)^{2}} & \text { Least Squares } \\
G_{r}(s)=\frac{14.63+14.19 s+1.032 s^{2}}{36.96+14.84+s^{2}} & \text { Non Linear Programming } \\
G_{r}(s)=\frac{0.1351+0.4075 s+1.052 s^{2}}{0.6078+1.935 s+s^{2}} & \text { NLP with equality constraint }
\end{array}
$$


The frequency response of the real system and the reduced models is illustrated by the (Figure 2)
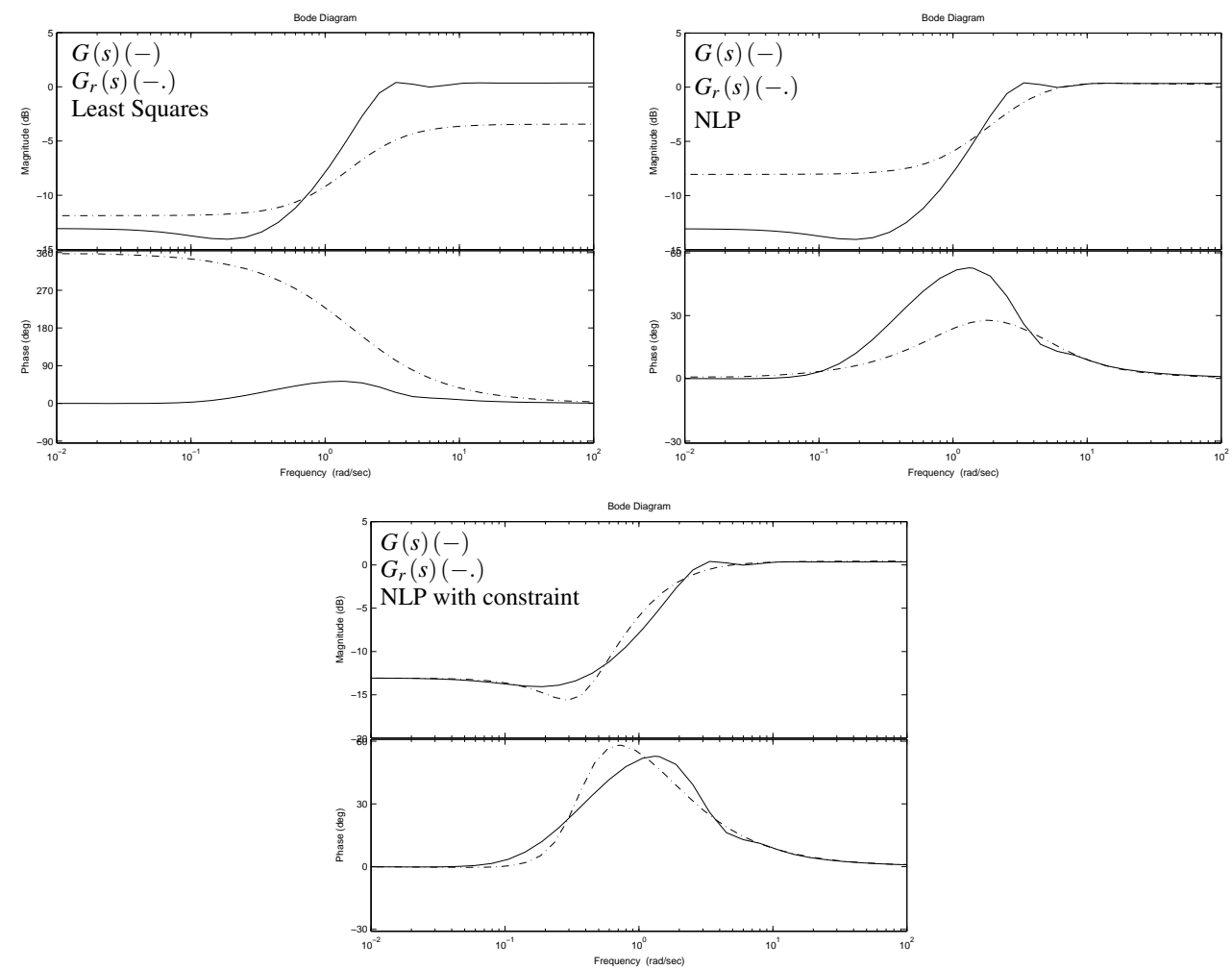

Figure 2: Frequency response of real system and reduced models

Remark 5. We saw that the moments may be used for model reduction; we presented three optimization methods: Least Squares, Non Linear Programming and Non Linear Programming with equality constraint. If the optimization is done in low frequencies, the Non Linear Programming can be used without equality constraint take into account the radius of convergence which allow to ensure the low frequencies behaviour.

\section{Summary and Conclusions}

In this paper, we presented a new method for model reduction and controller design. The technic is based on the moments tool which is able to give a description of any linear system or linear system with time delay in low frequencies using time moments or around a given frequency using the frequency moments.

The optimization procedure is composed of two steps, in the first one, we use least squares algorithm to have reduced model to initialize the non linear programming with equality constraint between the two first time moments between the reduced model and the full order system.

For controller design, the aim is to ensure the closed loop performances using a reference model which regroups dominant poles, auxiliary poles and system's singularities.

Using Youla parametrization, we obtain an ideal controller which will be reduced for implementation using the moments. 


\section{References}

[1] K.Astöm and B.Wittenmark "Computer Controlled Systems, Theory and Design", Prenctice Hall (1984)

[2] A.Bentayeb, N.Maamri and D.Mehdi "Moments Based Synthesis Approach Comparison with $\mathcal{H}_{\infty}$ Design",IFAC DECOM-TT "Istanbul, Turkey" (2003).

[3] S.Boyd and C.Barratt "Linear Controller Design-Limits of Performance". Prentice Hall (1991).

[4] K.V.Fernando and H.Nicholson "Singular perturbational model reduction of balanced systems", IEEE Transactions on Automatic Control, AC-27:2:466-468 (1982).

[5] N.Maamri and J.C.Trigeassou "PID design for time delayed systems by the method of moments", European Control Conference, Groningen Holland (1993).

[6] F.Monroux. "Méthodologie Générale de Synthèse de Correcteurs par la Méthode des Moments, Approche Mixte: Fréquentielle et Temporelle", Thèse de doctorat, Université de Poitiers, 1999.

[7] N.Maamri, A.Bentayeb and J-C.Trigeassou "Design and Iterative Optimization of Reduced Robust Controllers with Equality-Constraints" ROCOND-Milan (2003).

[8] D.W.Marquardt "An Algorithm for Least-Squares Estimation of Non Linear Parameters" Journal of Soc. Indust. Appl. Math V(11-2) (1963).

[9] M.G.Safonov and R.Y.Chiang "A schur method for balanced truncation model reduction". IEEE Transactions on Automatic Control AC-34:729-733, (1989).

[10] S.Skogested and I.Postlethwaite "Multivariable Feedback Control", JW (1996).

[11] M.S.Tombs and I.Postlethwaite "Truncated balanced realization of a stable non-minimal statespace system",International Journal of Control:46:1319-1330.

[12] J.C.Trigeassou "La méthode des moments en automatique". CIFA, Lille (2000).

A.Bentayeb, N.Maamri and J-C.Trigeassou

University of Poitiers

Laboratoire d'Automatique et d'Informatique Industrielle

40 Avenue du Recteur Pineau 86022 Poitiers

E-mail: abdelmadjid.bentayeb@gmail.com

Received: November 24, 2006

Editor's note about the author:

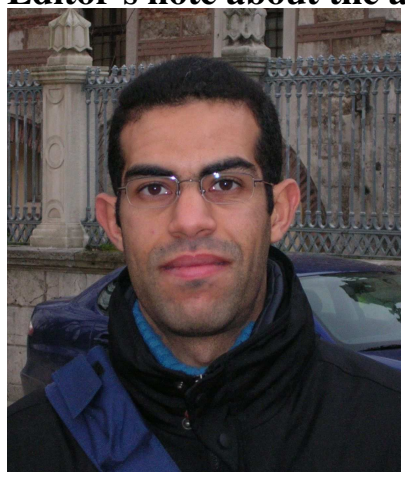

Abdelmadjid Bentayeb was born on August 9, 1977 in Annaba (Algeria). He received the engineer diploma of automatic control in 2000 and the Applied Studies Diploma of advanced control in 2001 at the University of Annaba. He received the Master degree of automatic and industrial data processing in 2002 and the PhD degree of automatic in 2006 with a very honourable distinction at the University of Poitiers. Currently, he prepares an industrial orientation of his work carried out at University of Poitiers. His current research interests include the multivariable robust control systems and model reduction using the method of the moments. 